\title{
Aplicación de la metodología STAR para el análisis del riesgo sísmico de la provincia Bolívar - Ecuador
}

\author{
Application of the STAR methodology for the analysis o \\ f the sismic risk of the province Bolivar - Ecuador
}

Anita Karina Serrano Castro. ${ }^{1}$, Juan Carlos Muyulema Allaica. ${ }^{2}$, Roberto Bernardo Usca Veloz. ${ }^{3}$ \& Martha Magdalena González Rivera. ${ }^{4}$

\begin{abstract}
.
DOI: https://doi.org/10.33262/cienciadigital.v3i4.968

Seismic risk is the probability of occurrence of a natural adverse event, associated with human and material losses, which is established by multiplying the threat by the threat; What is intended through this research is to identify the level of seismic risk of buildings in the Bolivar province, for this the Star methodology is used, which includes a heuristic, qualitative and quantitative study, which allows us to identify the level of sensitivity In addition, the threat level of the province which was achieved through the multiplication of the $\mathrm{Z}$ factor established in the Ecuadorian Construction Standards and the maximum magnitude of an earthquake through the incidence of geological faults that cross the Bolivar province, to establish the value of seismic risk. Therefore, a field work was carried out applying the vulnerability matrix for information gathering. In addition, the management of the computer program called ArcGIS was applied, which specified us to delimit the levels of vulnerability and seismic risk. As a result of the study carried out, it was determined that

${ }^{1}$ Universidad Estatal de Bolívar, Facultad Ciencias de la Salud y del Ser Humano, Escuela de Administración para Desastres y Gestión del Riesgo, Guaranda, Ecuador, anykarina15@gmail.com

2 Pontificia Universidad Católica del Ecuador Sede Ambato (PUCESA) Escuela de Diseño Industrial Ambato, Ecuador, juanca327@hotmail.com

${ }^{3}$ Universidad Estatal de Bolívar, Facultad de Ciencias de la Educación, Sociales, Filosóficas y Humanísticas, Guaranda, Ecuador, robtronics.rv17@gmail.com

${ }^{4}$ Universidad Estatal de Bolívar, Facultad Ciencias de la Salud y del Ser Humano, Escuela de Administración para Desastres y Gestión del Riesgo, Guaranda, Ecuador, marthy_1@yahoo.es
\end{abstract}


there is a medium level of threat and threat, thus giving as a provincial seismic risk a medium level; finally, it can be concluded that in the Bolívar province a great percentage their homes have a structural system of reinforced concrete, this reduces the threat to a seismic threat, however, for future constructions, the construction regulations of each country should be considered to avoid future disaster situations.

Keywords: Threat, Vulnerability, Seismic Risk, Geological Failures, Bolivar Province.

\section{Resumen.}

El riesgo sísmico es la probabilidad de ocurrencia de un evento adverso natural, asociado a las pérdidas humanas y materiales, que se establece mediante la multiplicación de la amenaza por la vulnerabilidad; lo que se pretende mediante esta investigación es identificar el nivel de riesgo sísmico de las edificaciones de la provincia Bolívar, para ello se utiliza la metodología STAR, que abarca un estudio Heurístico, cualitativo y cuantitativo, la misma que nos permite identificar el nivel de vulnerabilidad, además el nivel de amenaza de la provincia la cual se obtuvo mediante la multiplicación del factor $Z$ establecido en las Normas de Construcción Ecuatoriana y la magnitud máxima de un sismo por medio de la incidencia de las fallas geológicas que atraviesan la provincia Bolívar, para establecer el valor del riesgo sísmico. Por consiguiente, se realizó un trabajo de campo aplicando la matriz de vulnerabilidad para el levantamiento de información. Además, se aplicó el manejo del programa informático denominado ArcGIS el cual nos permitió delimitar los niveles de vulnerabilidad y riesgo sísmico. Como resultado del estudio realizado, se determinó que existe un nivel de vulnerabilidad y amenaza medio, dando, así como riesgo sísmico provincial un nivel medio; finalmente se puede concluir que en la provincia Bolívar un gran porcentaje de sus viviendas tienen un sistema estructural de hormigón armado, esto hace que disminuya la vulnerabilidad ante una amenaza sísmica, no obstante, para las futuras construcciones se deben considerar las normas de construcción de cada país para evitar futuros escenarios de desastres.

Palabras claves: Amenaza, Vulnerabilidad, Riesgo Sísmico, Fallas Geológicas, Provincia Bolívar.

\section{Introducción.}

El concepto de riesgo sísmico está siempre relacionado con la predicción de pérdidas futuras y está íntimamente ligado a la psicología personal o colectiva, razón por la que, obviamente, es difícil darle objetividad (Aledo \& Sulaiman, 2015; Montiel \& Negrete, 2017). Por este motivo es tan compleja la evaluación del riesgo, aun cuando se trate solamente de su dimensión física (Díaz, 2017). Además, es absolutamente necesario que el riesgo esté siempre asociado con la decisión, 
con la ejecución de una acción que se debe llevar a cabo (Porras, Linkimer, Arroyo, \& Taylor, 2018).

Ayala, Delgadillo, \& Ferrer (2017) describen a la amenaza sísmica, como peligro sísmico, esta se cuantifica en términos de los periodos de retorno de intensidades sísmicas. Según ZambranoHernández \& Gómez-Serna (2015) este se calcula de manera indirecta, evaluando primero la tasa de actividad sísmica en las fuentes generadoras de temblores, y después se integran los efectos que producen, en un sitio dado, los sismos que se generan en la totalidad de las fuentes. Por otro lado, Salgado-Gálvez, Carreño, Barbat, \& Cardona (2016) definen a la amenaza sísmica como un conjunto de escenarios estocásticos que permite considerar el efecto de eventos pequeños, moderados y extremos en las posibles pérdidas futuras.

Fernández (2017) manifiesta que la vulnerabilidad estructural se refiere a la susceptibilidad que la estructura presenta frente a posibles daños en aquellas partes del establecimiento hospitalario que lo mantienen en pie ante un sismo intenso. Esto incluye cimientos, columnas, muros, vigas y losas. Para Ayala, Delgadillo, \& Ferrer (2017) los sismos son movimientos vibratorios en el interior de la tierra que suben a la superficie en forma de ondas sísmicas provocando daños en las infraestructuras, bienes, servicios, recursos y por ende ocasionan perdidas tanto humanas como económicas.

Según Chunga (2016); Ayala, Delgadillo, \& Ferrer (2017); Benavides (2018); Amangadi, Yasuma, \& Barragán (2019) durante la segunda mitad del siglo XX hubo un rápido aumento a escala mundial de las pérdidas económicas y de vidas debido a la ocurrencia de desastres naturales. Esta tendencia se ha sostenido durante estos primeros años del siglo XXI, y los desastres ocasionados por terremotos no son la excepción; es alarmante observar la frecuencia con que se frecuentan los sismos de gran magnitud en diferentes partes del mundo y los costos en millones de dólares que generan. Así es el caso de los terremotos de Chile y México (1985), de Armenia (1988), California (1989 y 1994), Japón (1995, 2007, 2011, 2013, 2018, 2019), Turquía e India (1999), Irán (2003), de Haití 2010, Ecuador (2016, 2019) entre otros.

Ecuador se encuentra localizado en el cinturón de fuego del Océano Pacífico, al noroccidente de Sudamérica. Es uno de los países más densamente poblados de la región (Contreras, 2014). La actividad sísmica es causada principalmente por la subducción de la Placa Oceánica de Nazca y la presencia de un complejo sistema de fallas activas locales (Quinde \& Reinoso, 2016). Bajo la información disponible y dada la alta peligrosidad sísmica a la que se ve expuesta el Ecuador, que ha sido constatada por las pérdidas humanas y materiales documentadas en reportes históricos, especialmente la noche del 16 de abril de 2016. Con epicentro a $30 \mathrm{~km}$ de Pedernales, en la provincia de Manabí, un terremoto de magnitud 7,8 en la escala de Richter. A continuación, se presenta a manera de breve balance, algunos datos relevantes con el objeto de plasmar la magnitud de lo ocurrido. Las pérdidas humanas fueron eminentes, ascendiendo a 673; adicionalmente, la población directamente afectada se estimó en 85532 personas, mientras que otras 385000 sufrieron daños indirectos (Carrión, Giunta, Mancero, \& Jiménez, 2017). Así, se plantea la 
conveniencia de desarrollar nuevos estudios de riesgo sísmico que integren el estado del conocimiento actual y utilicen, además, metodologías recientes y acordes a la información disponible, con el fin de generar resultados actualizados sobre perdidas esperadas por terremotos futuros que ayuden en la oportuna toma de decisiones, gestión local y gubernamental en este trascendental campo.

Por otra parte, existen en la actualidad estructuras en las zonas sísmicas que no se diseñaron para resistir acciones dinámicas horizontales y, por tanto, fueron construidas sin seguir los requisitos de una norma de diseño sismorresistente e incluso, muchas veces, sin control de los materiales (Quinde \& Reinoso, 2016). En el proyecto de otras muchas estructuras se utilizaron normas que no cumplen criterios de diseño que hoy son generalmente aceptados (Beauval et al., 2013). Por todo ello, las variables asociadas a la estructura y a su respuesta pueden tener incertidumbres aún mayores. Según Amangadi, Yasuma, \& Barragán (2019) debido al mal ordenamiento y gestión territorial que existe en las ciudades e todas las veinte y cuatro provincias ecuatorianas, especialmente en las zonas urbanas en expansión se generan los diferentes niveles de riesgos dependiendo de la amenaza, esto también genera la poca preparación de la población para hacer frente a los diferentes tipos de desastres. Las recurrencias de activación de fallas capaces de generar sismos moderados a fuertes, puede estar en el orden de decenas a centenas de años, y en otras ocasiones cada 30.000 años. La historia sísmica puede ser complementada con datos paleo sísmicos, a través de los estudios de estructuras de deformaciones sinsedimentarias (Chunga, 2016).

La Provincia Bolívar está situada en la parte centro oeste del Ecuador ubicándose así en una zona sísmica de $4^{\circ}$ que representa alta peligrosidad según el Instituto Geofísico Militar esto se debe a que está rodeada de un sistema de fallas activas tanto regionales (falla de Pallatanga), como locales (falla del rio Chimbo y falla del rio Salinas), esto hace que la zona sea más susceptible a la ocurrencia de un sismo (Amangadi, Yasuma, \& Barragán, 2019).

Así, el objeto general de este documento es identificar el nivel de riesgo sísmico de las edificaciones de la provincia Bolívar, para ello se utiliza la metodología STAR, para lo cual fue necesario dar cumplimiento desde disímiles ópticas a un triple objetivo. El primero se centró en el cálculo de la amenaza sísmica, así como el peligro sísmico. El segundo busco calcular la vulnerabilidad. El tercero se enarcó en el cálculo de los factores de riesgo sísmico que potencialmente incurre la provincia de Bolívar.

\section{Metodología.}

En el presente estudio del riesgo sísmico se utilizó la metodología "STAR" que es un análisis técnico de tipo sísmico, contemplado desde el área de Gestión de Riesgos, necesario para complementar el estudio de vulnerabilidad estructurales realizado por un profesional en el área. Se ha seleccionado esta metodología ya que con este documento se pretende validar la misma puesto 
que en diversos estudios de análisis de riesgos sísmico, no se considera a la magnitud máxima de un sismo producido por una falla geológica incidente en la zona de estudio.

\section{Variables e indicadores para el cálculo de la amenaza sísmica, vulnerabilidad de estructuras y riesgo sísmico}

Partiendo de las características físicas de las edificaciones que se encuentran presentes en el catastro municipal, se definen aquellas que inciden directamente en el comportamiento estructural de la edificación frente a la amenaza considerada, para calificarla de manera cualitativa y, ponderar los resultados con el objetivo de encontrar un único valor o grado de vulnerabilidad para cada edificación, así como también para la amenaza sísmica y el riesgo sísmico. Con la metodología planteada (STAR), los valores y pesos, para la calificación y ponderación, se deducen de acuerdo con el conocimiento del comportamiento estructural de las diferentes tipologías de edificaciones, frente a la amenaza determinada, utilizando como modelo las experiencias documentadas en desastres pasados y, con la información proveniente de la literatura técnica reciente a nivel mundial.

Para realizar el cálculo de la amenaza los indicadores y variables se presentó la Tabla 3 con el indicador factor $\mathrm{Z}$ y sus respectivas variables la aceleración de la honda sísmica en la roca y en la Tabla 4 con el indicador Máxima magnitud liberada por la falla con sus indicadores ecuación general, falla inversa y falla transcurrente; en donde para poder aplicar cualquiera de las 3 variables se debe conocer la longitud en $\mathrm{km}$ de la falla geológica sujeta a evaluación.

Para realizar el cálculo de la vulnerabilidad se presentan en la Tabla 6 con los indicadores edad de la edificación y sus variables (menor a 4 años, de 4 a 40 años y mayor de 40 años), número de pisos (1 piso, de 2 a 3 pisos y mayor a 3 pisos), tipo de construcción (concreto, prefabricado, mixto y madera), mantenimiento (si y no), reforzamiento (si y no).

Finalmente, el cálculo del riesgo se lo hace mediante el remplazo de los valores obtenidos en los cálculos de la amenaza y vulnerabilidad en la fórmula del riesgo obteniendo niveles de riesgo entre 0,02 y 3 caracterizándose como medio, alto y muy alto (Tabla 8).

La zona de estudio es la provincia de Bolívar misma que se encuentra ubicada en la parte centro occidental interandina, ocupa la hoya del rio Chimbo y se encuentra entre las siguientes coordenadas geográficas:

\section{Coordenadas UTM zona 17 sur}

- 9872857 hasta 9756351 metros Norte

- 678594 hasta 742762 metros Este 


\section{Población y muestra}

Con relación a la población, según el Instituto Nacional de Estadística y Censos - INEC (2010) la provincia Bolívar cuenta con 183641 habitantes, como se muestra en la siguiente Tabla 1. Como referencia adicional se tiene que, dentro de cada vivienda habitan por lo menos 5 personas. Deduciendo así que la provincia está compuesta por una infraestructura de 36728 viviendas.

Tabla 1: Población por cantones de la provincia Bolívar

\begin{tabular}{cccccc}
\hline \multicolumn{5}{c}{ Población por cantones de la provincia Bolívar } \\
\hline No. & CANTÓN & POBLACIÓN & PORCENTAJE & \# VIVIENDAS & PORCENTAJE \\
\hline $\mathbf{1}$ & Caluma & 13129 & $7.15 \%$ & 2626 & $7.15 \%$ \\
\hline $\mathbf{2}$ & Chillanes & 17406 & $9.48 \%$ & 3481 & $9.48 \%$ \\
\hline $\mathbf{3}$ & Echeandía & 12114 & $6.60 \%$ & 2423 & $6.60 \%$ \\
\hline $\mathbf{4}$ & Guaranda & 91877 & $50.03 \%$ & 18375 & $50.03 \%$ \\
\hline $\mathbf{5}$ & Las Naves & 6092 & $3.32 \%$ & 1218 & $3.32 \%$ \\
\hline $\mathbf{6}$ & Chimbo & 15779 & $8.59 \%$ & 3156 & $8.59 \%$ \\
\hline $\mathbf{7}$ & San Miguel & 27244 & $14.84 \%$ & 5449 & $14.84 \%$ \\
\hline & TOTAL & $\mathbf{1 8 3 6 4 1}$ & $\mathbf{1 0 0 . 0 0 \%}$ & $\mathbf{3 6 7 2 8}$ & $\mathbf{1 0 0 . 0 0 \%}$
\end{tabular}

Fuente: Los autores basados en INEC (2010).

El cálculo de la muestra se hará en base a un universo finito, es decir conocemos el total de la población y deseamos saber cuántos del total tendremos que estudiar. Según Álvarez (2012), para calcular el tamaño de la muestra suele utilizarse la siguiente formula:

Fórmula:

$$
n=\frac{Z^{2} P Q N}{Z^{2} P Q+N e^{2}}
$$

Fuente: (Álvarez, 2012)

\section{Donde:}

$\mathrm{n}=$ Tamaño de la muestra

$\mathrm{Z}=$ Nivel de confianza (1.96)

$\mathrm{N}=$ Población (36 728)

$\mathrm{P}=$ Probabilidad de éxito $(50 \%)$

$\mathrm{Q}=$ Probabilidad de fracaso $(50 \%)$

$\mathrm{e}=$ error $(0.05)$ 


\section{Cálculo de la muestra:}

$$
\begin{gathered}
\mathrm{n}=\frac{(1.96)^{2}(0.50)(0.50) 36728}{(1.96)^{2}(0.50)(0.50)+36728(0.05)^{2}} \\
\mathrm{n}=\frac{35273.5712}{0.9604+91.82} \\
\mathrm{n}=380
\end{gathered}
$$

El total de la muestra a estudiar corresponde a 380 viviendas de la provincia de Bolívar. Las cuáles serán evaluadas para identificar las amenazas, vulnerabilidad y riesgo sísmico de las mismas.

Para la realización del trabajo de campo se procedió al cálculo del tamaño de la muestra de viviendas por cantones de la provincia Bolívar, en base a la estratificación de la muestra, comparativamente como se expone en la Tabla 2.

Tabla 2: Estratificación de la muestra de viviendas por cantones de la provincia de Bolívar.

Tamaño de la muestra de viviendas por cantones de la provincia Bolívar No. CANTÓN \# VIVIENDAS PORCENTAJE TAMAÑO DE

\section{LA MUESTRA}

\begin{tabular}{ccccc}
\hline $\mathbf{1}$ & Caluma & 2626 & $7.15 \%$ & 27 \\
\hline $\mathbf{2}$ & Chillanes & 3481 & $9.48 \%$ & 36 \\
\hline $\mathbf{3}$ & Echeandía & 2423 & $6.60 \%$ & 25 \\
\hline $\mathbf{4}$ & Guaranda & 18375 & $50.03 \%$ & 190 \\
$\mathbf{5}$ & Las Naves & 1218 & $3.32 \%$ & 13 \\
\hline $\mathbf{6}$ & Chimbo & 3156 & $8.59 \%$ & 33 \\
\hline $\mathbf{7}$ & San Miguel & 5449 & $14.84 \%$ & 56 \\
\hline & TOTAL & $\mathbf{3 6 ~ 7 2 8}$ & $\mathbf{1 0 0 . 0 0 \%}$ & $\mathbf{3 8 0}$ \\
\hline
\end{tabular}

Fuente: Los autores basados en INEC (2010) y el cálculo de la muestra.

\section{Resultados.}

La presencia de varias fallas Geológicas próximas y en la provincia de Bolívar son una amenaza latente puesto que son consideras activas, por otro lado, la vulnerabilidad estructural es un factor determinante a la hora de contrarrestar una amenaza sísmica; y más aun a la hora de disminuir el nivel de riesgo adyacente a la actividad sísmica, los resultados obtenidos son los siguientes: 


\section{Cálculo de la amenaza.}

La amenaza es definida como la probabilidad de ocurrencia de un evento potencialmente desastroso durante cierto periodo de tiempo en un sitio dado.

La Tabla 3 determina el Fz provincial el cual se realizó un promedio de los valores del Fz de cada una de las cabeceras cantonales obteniendo como resultado un valor de 0,33 que corresponde a una zona sísmica alta por ende tiene una ponderación de 2 "ALTA”.

Tabla 3: Cálculo de la aceleración máxima en roca esperada para un sismo. (Factor Z)

Cálculo de la aceleración máxima en roca esperada para un sismo. (Factor Z)

\begin{tabular}{|c|c|c|c|c|c|c|}
\hline \multicolumn{7}{|c|}{$A=\frac{\mathbf{F}(\mathbf{Z}) * \mathbf{M} \text { máx. }}{10}$} \\
\hline Zona sísmica & I & II & III & IV & $\mathbf{V}$ & VI \\
\hline Valor factor $\mathbf{Z}$ & 0,15 & 0,25 & $\mathbf{0 , 3 0}$ & 0,35 & 0,40 & $\geq \mathbf{0 , 5 0}$ \\
\hline $\begin{array}{c}\text { Caracterización del peligro } \\
\text { sísmico }\end{array}$ & Intermedia & Alta & Alta & Alta & Alta & Muy Alta \\
\hline Ponderación de la metodología & 1 & 2 & 2 & 2 & 2 & 3 \\
\hline Marcar con una X la ponderación & & & & $\mathbf{X}$ & & \\
\hline
\end{tabular}

Fuente: Elaborado propia.

La Tabla 4 establece la M-máx. probable de un sismo, por lo cual se ha realizado una media de las 15 fallas que son más cercanas y con una mayor incidencia tectónica sobre la Provincia de Bolívar obteniendo como resultado una M-máx. de 6,82 con una ponderación de 3 siendo su caracterización de la magnitud "ALTA". Como no se obtuvo mayores datos acerca de las fallas se determinó la M-máx. Con le ecuación general.

Tabla 4: Cálculo de la máxima magnitud de un sismo debido a las características de una determinada falla

\section{M-máx. probable de un sismo}

\begin{tabular}{cccc}
\hline \multicolumn{2}{c}{ Ecuación general } & M máx. $=4,86+1,33 \times \log \mathrm{L}$ & $\mathbf{X}$ \\
\hline Falla & Foco profundo & M máx. $=5,47+1,0 \times \log \mathrm{L}$ \\
\cline { 2 - 3 } inversa & Foco superficial & M máx. $=6,04+0,79 \times \log \mathrm{L}$ \\
\hline
\end{tabular}




\begin{tabular}{cccc}
\hline Falla transcurrente & M máx. $=6,04+0,74$ x Log L & \\
\hline Magnitud & $\begin{array}{c}\text { Caracterización de la } \\
\text { magnitud (Mw) }\end{array}$ & $\begin{array}{c}\text { Ponderación de la } \\
\text { metodología }\end{array}$ & $\begin{array}{c}\text { Marcar con } \\
\text { una X la } \\
\text { ponderación }\end{array}$ \\
\hline $0,1-3,9$ & Baja & 1 & \\
\hline $4,0-6,0$ & Media & 2 & $\mathbf{X}$ \\
\hline $6,1-8,0$ & Alta & 3 & \\
\hline$>8,1$ & Muy alta & 4 & \\
\hline
\end{tabular}

Fuente: Elaborado propia.

En la Tabla 5 se ha determinado una amenaza sísmica MEDIA para la provincia de Bolívar, ya que al momento de aplicar la ecuación de la amenaza se ha obtenido un resultado de 0,6 equivalente a dicha caracterización.

Tabla 5: Resultado para el cálculo de la amenaza.

\begin{tabular}{|c|c|}
\hline \multicolumn{2}{|r|}{ Resultado para el cálculo de la amenaza } \\
\hline & $\boldsymbol{A}=\frac{2 * 3}{10}=0,6$ \\
\hline $\begin{array}{c}\text { Resultado de la } \\
\text { Amenaza }\end{array}$ & Caracterización de la AmenazaValor de la ecuación de la Amenaza \\
\hline $0,1-0,3$ & Bajo \\
\hline $0,4-0,6$ & Medio \\
\hline $0,7-0,9$ & Alto \\
\hline $1,0-1,2$ & Muy alta \\
\hline
\end{tabular}

Fuente: Elaborado propia.

La Figura 1 muestra las ubicaciones, las fallas, y las tasas de actividad de las principales funciones relacionadas con los terremotos, así como también actividad relacionada con fallas y pliegues de la era cuaternaria. 
Figura 1: Base de datos y mapa de fallas cuaternarias y pliegues de Ecuador y sus regiones en alta mar.

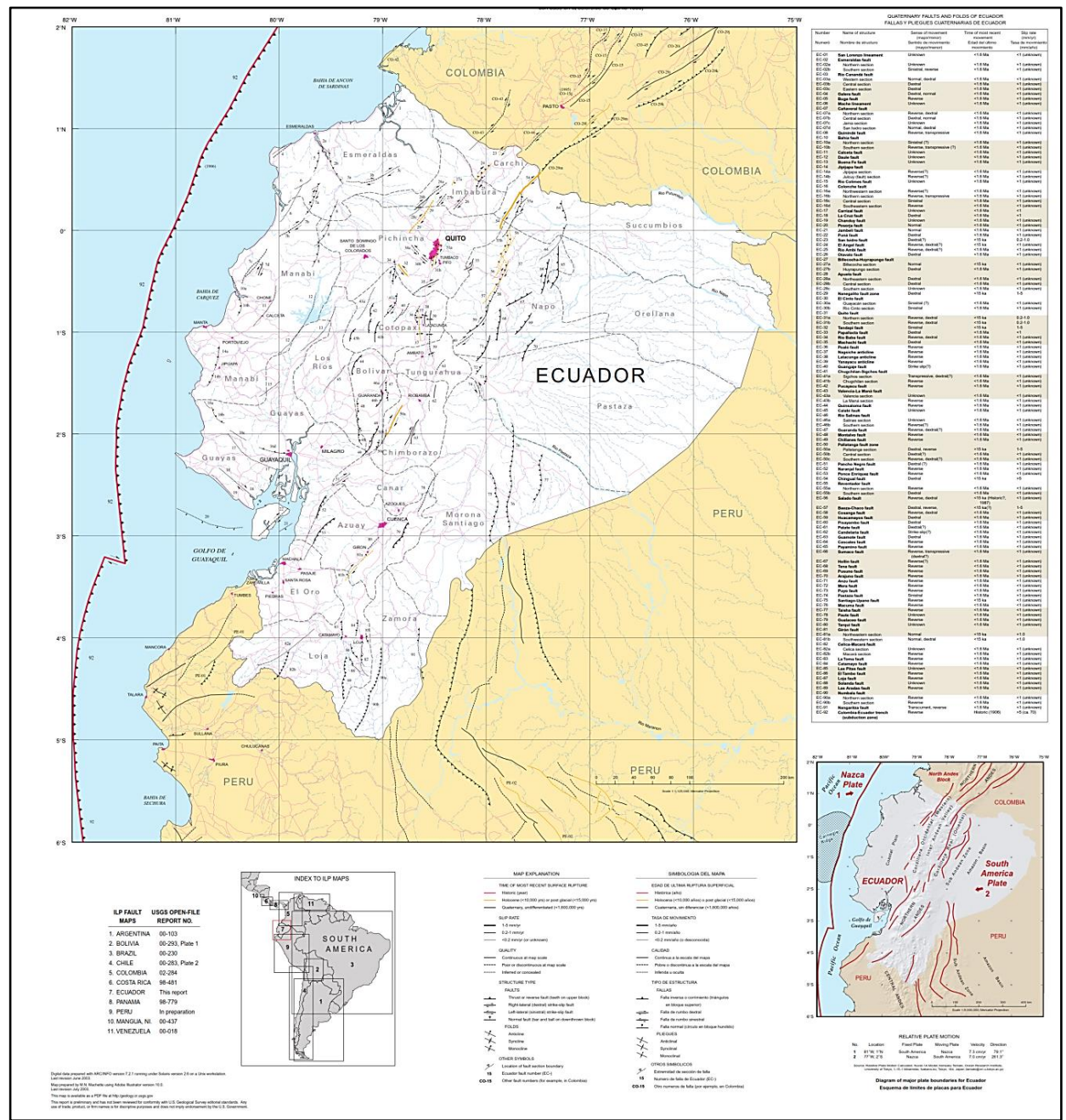

Fuente: Eguez (2013).

La Provincia Bolívar está rodeada por 14 fallas geológicas tanto inversas como normales, las mismas que nos permite realizar el estudio sobre el análisis de riesgos sísmicos y obtener el grado de la vulnerabilidad de cada cantón del mismo, a continuación, se describen las fallas geológicas con incidencia en la provincia, como se expone en la Figura 2. 
Figura 2: Base de datos y mapa de fallas cuaternarias y pliegues de Ecuador y sus regiones en alta mar.

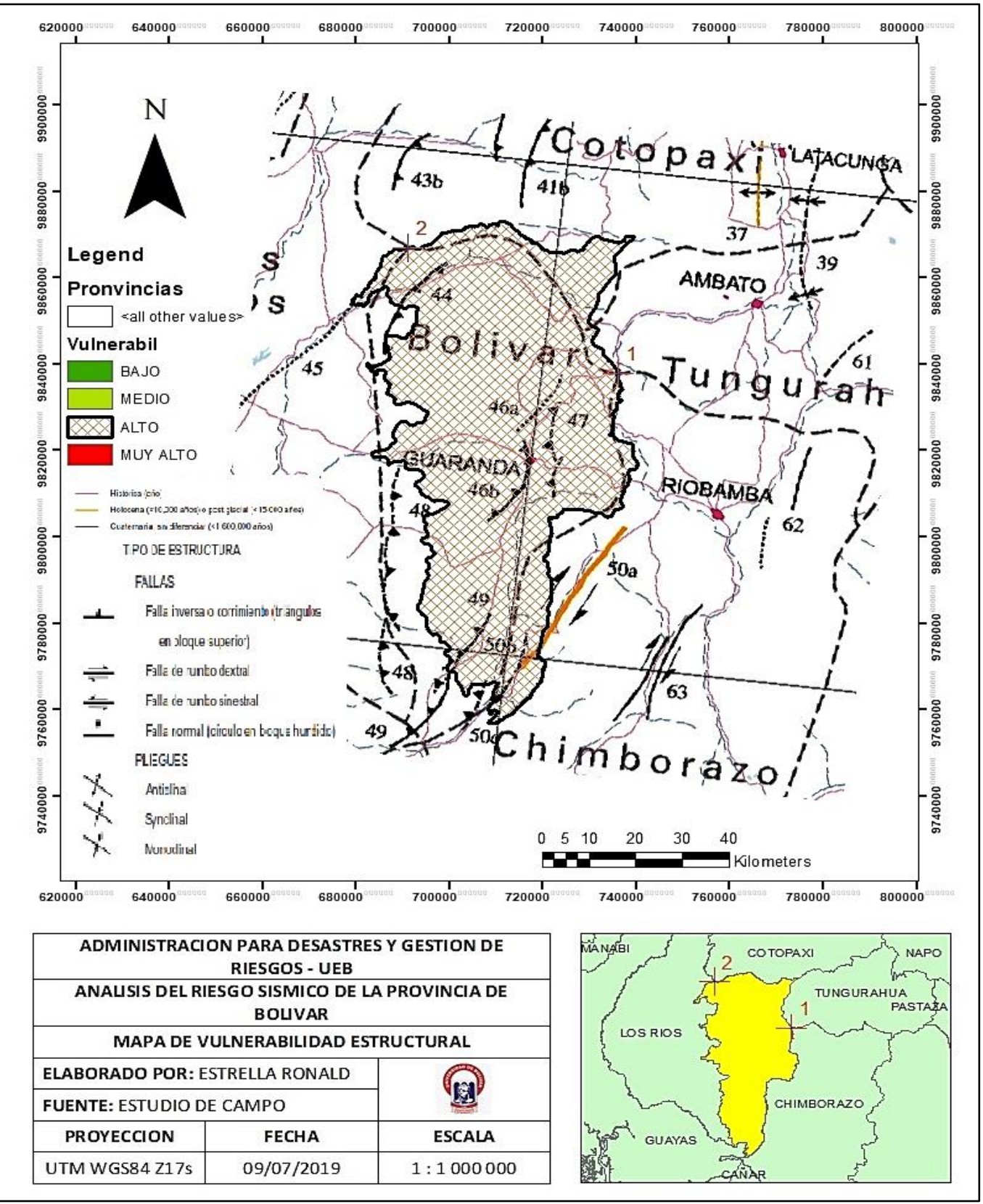

Fuente: Los autores y estudiante de apoyo al grupo investigador.

Fallas Geológicas con incidencia en la provincia de Bolívar y su M máx. 
Según Eguez (2013) existen 13 fallas geológicas con incidencia en la provincia de Bolívar y son:

1. Buena fe $(7,2)$

2. Nagshiche $(6,7)$

5. Chugchilan $(6,3)$

6. Quinsaloma $(6,7)$

7. Calabi $(6,9)$

8. Rio salinas $(7,1)$

8.1. Sección norte

8.2. Sección sur

9. Guaranda $(6,4)$

14. 6)

15. Guamote $(6,8$
3. Latacunga $(7,1)$

4. La mana $(6,6)$

10. Montalvo $(6,5)$

11. Chillanes $(6,9)$

12. Pallatanga $(7,1)$

12.1. Sección norte

12.2. Sección central

12.3. Sección sur

13. Candelaria $(6$,

\section{Cálculo de la vulnerabilidad.}

La vulnerabilidad es como el grado de pérdida de un elemento o grupo de elementos bajo riesgo, resultado de la población ocurrencia de un evento desastroso, expresada en una escala que va de 0 , o sin daño, a 1 o pérdida total.

En la Tabla 6 se ha realizado la evaluación de la vulnerabilidad estructural a 380 viviendas de la Provincia de Bolívar ubicadas dentro del perímetro urbano, distribuidas equitativamente según el porcentaje obtenido de muestras totales de familias que habitan en esta zona.

Tabla 6: Ficha de evaluación para el cálculo de la vulnerabilidad

\begin{tabular}{|c|c|c|c|}
\hline \multicolumn{4}{|c|}{ Ficha de evaluación para el cálculo de la vulnerabilidad } \\
\hline $\begin{array}{l}\text { Ecu } \\
=(\end{array}$ & $\begin{array}{l}\text { ón para el cálculo de } \\
\qquad \begin{array}{l}\text { di } * \text { Pisos })+(\text { T.con } \\
10\end{array}\end{array}$ & $\begin{array}{l}\text { la Vulnerabilida } \\
* \text { Mant } * \text { Refor) } \\
\end{array}$ & $=V$ \\
\hline $\begin{array}{l}\text { Edad de la } \\
\text { edificación }\end{array}$ & $\begin{array}{c}\text { Caracterización de la } \\
\text { edad de la } \\
\text { edificación }\end{array}$ & $\begin{array}{c}\text { Ponderación de la } \\
\text { metodología }\end{array}$ & $\begin{array}{c}\text { Vulnerabilidad } \\
\text { Cantonal }\end{array}$ \\
\hline$<4$ años & Medio & 1 & \\
\hline $4-40$ años & Alto & 2 & Caluma \\
\hline$>40$ años & Muy alto & 3 & \\
\hline
\end{tabular}


Vol. 3, Nº4, p. 227-249,octubre - diciembre, 2019

\begin{tabular}{|c|c|c|c|}
\hline Número de Pisos & $\begin{array}{c}\text { Caracterización de la } \\
\text { edad de la } \\
\text { edificación }\end{array}$ & $\begin{array}{c}\text { Ponderación de la } \\
\text { metodología }\end{array}$ & $\begin{array}{l}\text { Chillanes } \\
1,06\end{array}$ \\
\hline 1 piso & Medio & 1 & \\
\hline 2-3 pisos & Alto & 2 & \multirow{3}{*}{$\begin{array}{c}\text { Echeandia } \\
0,84\end{array}$} \\
\hline$>3$ pisos & Muy alto & 3 & \\
\hline $\begin{array}{c}\text { Tipo de } \\
\text { construcción }\end{array}$ & $\begin{array}{l}\text { Caracterización del } \\
\text { tipo de construcción }\end{array}$ & $\begin{array}{c}\text { Ponderación de la } \\
\text { metodología }\end{array}$ & \\
\hline Concreto & Bajo & 1 & \multirow{3}{*}{$\begin{array}{c}\text { Guaranda } \\
0,87\end{array}$} \\
\hline Prefabricado & Medio & 2 & \\
\hline Mixto & Alto & 3 & \\
\hline Madera & Muy alto & 4 & \multirow{2}{*}{$\begin{array}{c}\text { Las Naves } \\
0,65\end{array}$} \\
\hline Mantenimiento & $\begin{array}{l}\text { Caracterización de } \\
\text { mantenimiento }\end{array}$ & $\begin{array}{c}\text { Ponderación de la } \\
\text { metodología }\end{array}$ & \\
\hline $\mathrm{Si}$ & Medio & 1 & \multirow{3}{*}{$\begin{array}{c}\text { San Miguel } \\
0,93\end{array}$} \\
\hline No & Alto & 2 & \\
\hline Reforzamiento & $\begin{array}{l}\text { Caracterización de } \\
\text { reforzamiento }\end{array}$ & $\begin{array}{c}\text { Ponderación de la } \\
\text { metodología }\end{array}$ & \\
\hline $\mathrm{Si}$ & Medio & 1 & Chimbo \\
\hline No & Alto & 2 & 1,02 \\
\hline
\end{tabular}

Fuente: Elaborado propia.

En la Figura 3 se puede observar el estudio de la vulnerabilidad estructural de los 7 cantones de la provincia se obtuvo un nivel medio, dando constancia que el Cantón Las Naves es el único con un nivel bajo del mismo.

Figura 3: Vulnerabilidad estructural de la provincia de Bolívar, nivel cantonal.

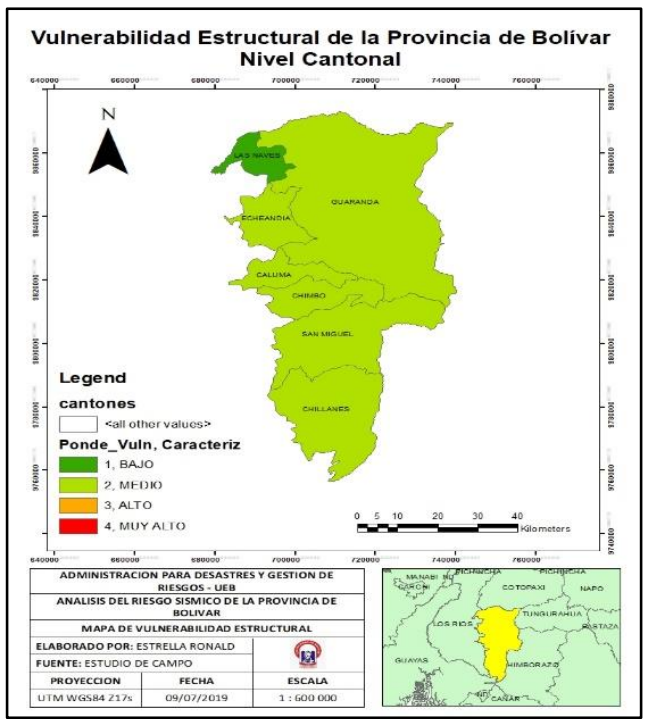

Fuente: Los autores y estudiante de apoyo al grupo investigador. 
En la Tabla 7 se presenta la significancia del resultado del cálculo de la vulnerabilidad provincial con un grado de certeza de valor explicativo, dato determinado a través de la obtención de una media del cálculo de la vulnerabilidad de cada cantón (Tabla 6), consiguiendo como resultado un valor de 0,95 , el cual se sitúa entre los rangos 0,7 - 1,3, lo que corresponde a una caracterización de la vulnerabilidad "MEDIA.

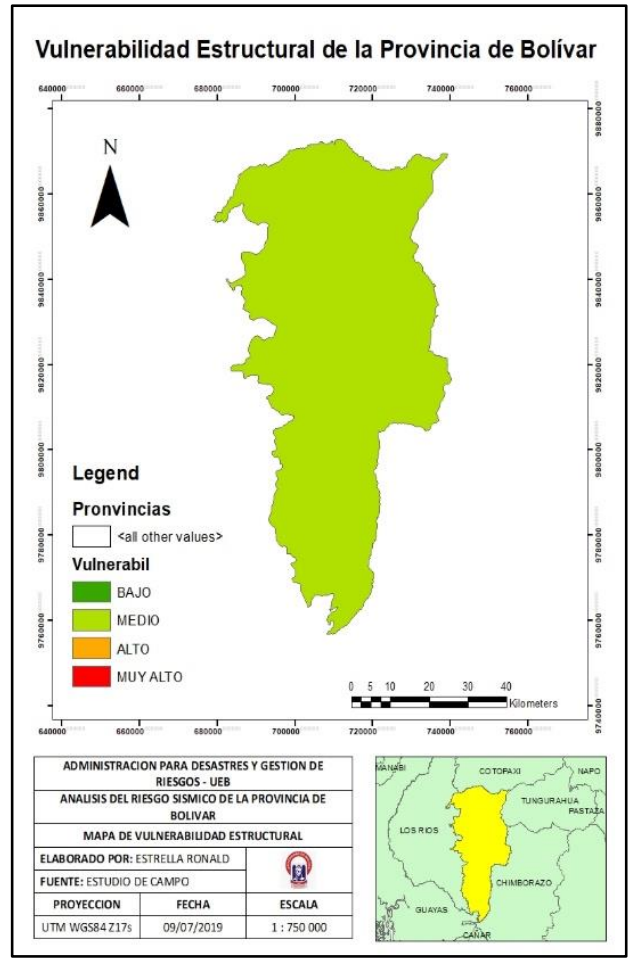

Tabla 7: Resultado para el cálculo de la vulnerabilidad

\begin{tabular}{ccc}
\hline \multicolumn{3}{c}{ Resultado para el cálculo de la vulnerabilidad } \\
\hline $\begin{array}{c}\text { Resultado de la } \\
\text { vulnerabilidad }\end{array}$ & $\begin{array}{c}\text { Caracterización de la } \\
\text { vulnerabilidad }\end{array}$ & $\begin{array}{c}\text { Valor de la ecuación de la } \\
\text { vulnerabilidad }\end{array}$ \\
\hline $0,2-0,6$ & Bajo & \\
\hline $0,7-1,3$ & Medio & $\mathbf{X}$ \\
\hline $1,4-2,0$ & Alto & \\
\hline $2,1-2,5$ & Muy alta \\
\hline
\end{tabular}

Fuente: Elaborado propia.

Después del estudio realizado dentro de la Provincia de Bolívar se obtuvo como resultado general un nivel medio de la vulnerabilidad estructural, donde se evidencia las viviendas mayores a 40 años en diferentes cantones, tal y como se muestra en el siguiente Figura 4. 
Figura 4: Vulnerabilidad estructural de la provincia de Bolívar.

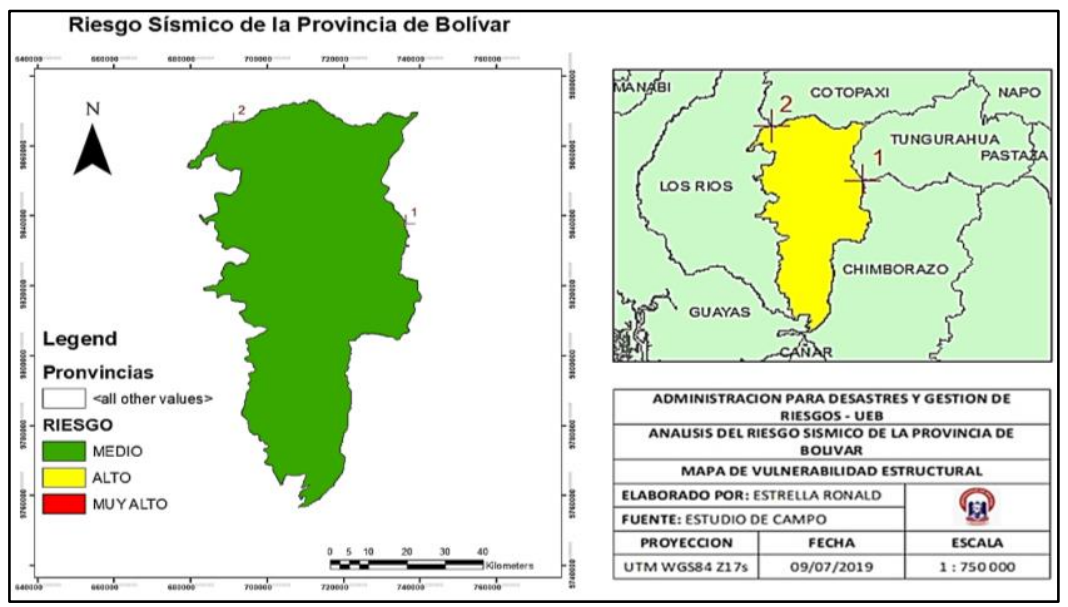

Fuente: Elaboración propia.

\section{Cálculo del riesgo.}

El riesgo es como el grado de pérdidas esperadas debido a la ocurrencia de un evento particular y como una función de la amenaza y la vulnerabilidad.

En la siguiente Tabla 8 se ha determinado un nivel de riesgo sísmico "Medio" para la provincia de Bolívar, obteniendo un resultado de 0,6, el cual está dentro de la ponderación $0,02-1,00$ equivalente a dicha caracterización.

Tabla 8: Caracterización del riesgo sísmico

\section{Caracterización del riesgo sísmico}

\begin{tabular}{ccc}
\hline Ecuación para el cálculo del Riesgo & $=\quad \boldsymbol{R}=A * V$ \\
& $\begin{array}{c}\boldsymbol{R}=0,6 * 0,95 \\
\\
\boldsymbol{R}=0,57\end{array}$ \\
\hline $\boldsymbol{R}=0,6 * 0,95$ & Caracterización del riesgo & Valor de la ecuación del \\
& sísmico & Riesgo \\
\hline $\boldsymbol{R}=0,57$ & Medio & 0,57 \\
\hline $1,01-2,00$ & Alto & \\
\hline $2,01-3,00$ & Muy Alto \\
\hline
\end{tabular}

Fuente: Elaborado propia.

Complementariamente a la caracterización del riesgo sísmico se ha aplicado la metodología STAR en los 7 cantones de la provincia de Bolívar, en general se obtuvo un resultado, un nivel medio ante los riesgos sísmicos, a pesar que la provincia se halla rodeada por diferentes fallas geológicas, tal y como se muestra en el siguiente Figura 5. 


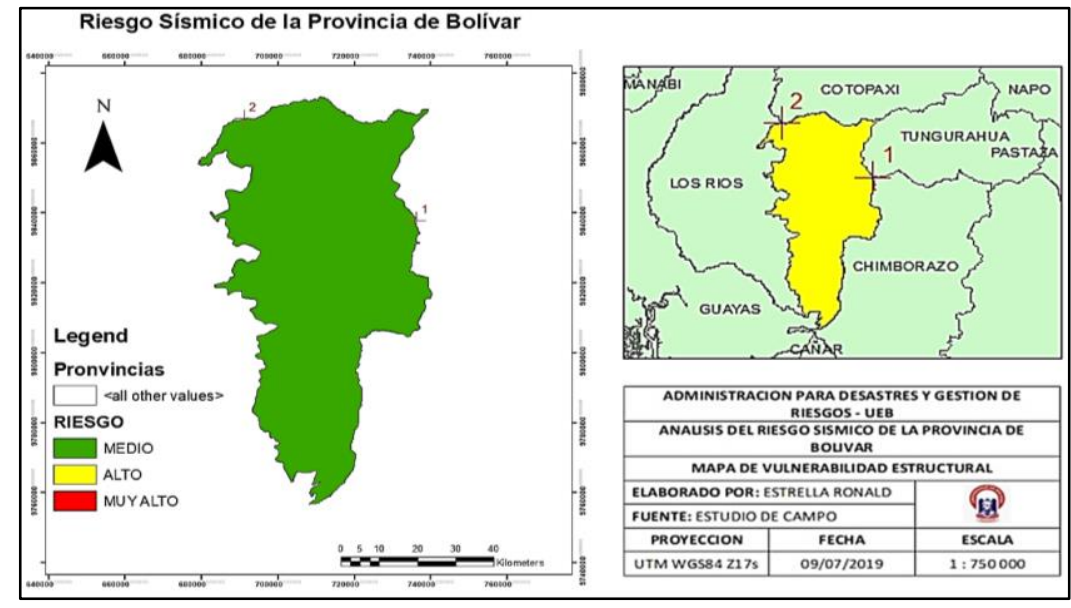

Figura 5: Riesgo sísmico de la provincia de Bolívar.

Fuente: Los autores y estudiante de apoyo al grupo investigador.

\section{Discusión.}

Los avances que se efectúan continuamente en el diseño de estructuras se emplean básicamente a las estructuras nuevas y en menor régimen a la rehabilitación de estructuras existentes (Castro-Pimienta et al., 2019). Sin embargo, el número total de estructuras existentes es muy grande en balance con el número de estructuras en construcción (VidaudQuintana, Frómeta-Salas, \& Vidaud-Quintana, 2019). Consiguientemente, para reducir las pérdidas debidas a los sismos, es ineludible mejorar también el comportamiento de las estructuras antiguas. Tal mejora demanda una evaluación previa de su comportamiento sísmico esperado, lo que sustenta las bases de los estudios de vulnerabilidad sísmica (Ayala, Delgadillo, \& Ferrer, 2017). Éstos estudios están orientados hacia la predicción de daños esperados en estructuras, en el supuesto de que suceda un terremoto de una cierta severidad (Jiménez, Cabrera, Sánchez, \& Avilés, 2018). A partir de dicha predicción, pueden precisarse soluciones de disminución de la vulnerabilidad estructural y, en consecuencia, de las pérdidas esperadas, es decir del riesgo sísmico.

En los últimos años se ha pretendido definir el riesgo sísmico para poder efectuar su gestión a fin de reducir los desastres económicos, sociales y ambientales que se puedan producir (Díaz, 2017). Para estimar el riesgo es preciso tener en cuenta, desde un punto de vista multidisciplinar (mezcla no-integradora de varias disciplinas en la que cada disciplina conserva sus métodos y suposiciones sin cambio o desarrollo de otras disciplinas), no solamente el daño físico esperado, el número de víctimas o las pérdidas económicas, sino también factores sociales, organizacionales e institucionales, concernientes con el desarrollo de las comunidades (Fernández, 2017; Martínez, 2018). A escala urbana, por ejemplo, la vulnerabilidad a modo de factor interno de riesgo debe relacionarse no simplemente con la 
exposición del contexto material o su susceptibilidad física de ser afectado, sino además con las fragilidades sociales y la falta de resiliencia de la comunidad expuesta (ZambranoHernández \& Gómez-Serna, 2015). La falta de una conveniente organización institucional y comunitaria, las extenuaciones en los preparativos para la atención de emergencias, la inestabilidad política y económica de un área geográfica favorecen a tener un mayor riesgo (Jiménez et al., 2018). Por lo tanto, las potenciales consecuencias no sólo están relacionadas con el impacto del acontecimiento, sino además con la capacidad para soportar el impacto y las implicaciones del mismo en el área geográfica afectada.

Según Beauval et al., (2013); Ordoñez (2016); Jiménez et al., (2018); Amangadi, Yasuma, \& Barragán (2019) en el Ecuador, tanto a nivel provincial como cantonal no se cumple a cabalidad las normas de construcción ecuatoriana vigentes desde el 2015, esto incide en el aumento de la vulnerabilidad física de edificaciones ante movimientos telúricos, sin embargo, las construcciones recientes y las regularidades en planta, así mismo como el tipo de suelo estable disminuye la exposición estructural ante dicho evento; por lo que se debe seguir trabajando para controlar las futuras construcciones.

El terremoto del 16 de abril de 2016, de magnitud 7.8, colapsó alrededor de 40 edificios de hormigón armado, ubicados en el centro de Portoviejo, con dolorosas pérdidas de vidas y un cuantioso perjuicio económico. Varias son las causas que llevaron al colapso a estos edificios, lo que se analiza en el presente artículo, entre las que se destacan: 1) la magnitud del sismo reflejada en los espectros de respuesta, obtenidos en la ciudad de Portoviejo, que para el rango de periodos que están alrededor de 0.5 segundos superó notablemente a los espectros que prescriben el Código Ecuatoriano de la Construcción del 2000, y las Normas Ecuatorianas de la Construcción de 2011 y 2015; 2) construcción de nuevos pisos sobre los ya existentes sin reforzar las estructuras; 3) estructuras bastante flexibles que tuvieron grandes desplazamientos 4) la tipología estructural que obliga a que las construcción tengan la planta baja con una altura de $5 \mathrm{~m}$ y mezzanines, y 5) amplificación de las ondas sísmicas por efecto de sitio. (Aguilar \& Mieles, 2016)

El terremoto con epicentro en Pedernales cambio la historia de la Gestión de Riesgos a nivel nacional, por lo que la Metodología STAR es una nueva alternativa para realizar nuevos estudios y de esta manera tomar las mejores decisiones respecto a la reducción de la vulnerabilidad física en edificaciones ante amenazas sísmicas

Por otro lado Ordoñez (2016) ha desarrollado un análisis de vulnerabilidad sísmica y riesgo de pérdida en las edificaciones en Quito, ante la ocurrencia de un evento sísmico. Como muestra representativa se toma el Centro Histórico, pues sus edificaciones comprenden las diferentes tipologías de construcción. Se utilizan metodologías probadas en Europa y Estados Unidos como HAZUS y PERPETUATE, y modelos desarrollados en Ecuador para la determinación de curvas de capacidad y fragilidad, puntos de desempeño y derivas máximas de piso. La muestra (2606 edificaciones) se clasifica con la metodología HAZUS. Para 
mampostería portante se utiliza el principio de similitud con el proyecto PERPETUATE. Como resultado se obtiene que, ante un evento desastroso, estas edificaciones sufrirían daño. Para la tipología de hormigón armado, se aplica la metodología HAZUS adaptada a Ecuador. Se calculan curvas de capacidad, puntos de desempeño y derivas de piso. Estos datos acceden a curvas de fragilidad que definen porcentajes de daño y vulnerabilidad. La ciudad de Quito tiene un alto grado de vulnerabilidad, no solo por las características propias del suelo y sus fallas, sino también, por la forma en que se diseña y construye. Los resultados no son concluyentes y son susceptibles de ser mejorados en función de la tecnología disponible.

Adicionalmente Ordoñez (2016) expresa que las ciudades más modernas en el Ecuador están situadas en áreas de peligrosidad sísmica moderada o baja, tienden a minusvalorar el peligro sísmico y a descuidar precauciones básicas de protección frente a los terremotos. Ello conlleva un aumento de su vulnerabilidad y, en consecuencia, suelen presentar un elevado riesgo sísmico que, a su vez, aumenta con el tiempo (Quinde \& Reinoso, 2016). Bajo este contexto, este trabajo probablemente está entre los primeros que obtienen escenarios de daño sísmico de una provincia, mediante una aproximación que permite y efectúa un análisis edificio a edificio.

La provincia Bolívar debido a su posición geográfica está atravesada por una serie de fallas geológicas y algunas de estas se ubican cerca de la provincia por lo que inciden en la generación de los sismos, por ello se evidencia la significativa necesidad de evaluar la vulnerabilidad física en edificaciones para evitar los riesgos de desastres en un futuro.

Hoy por hoy no se puede predecir los sismos por lo cual genera una gran incertidumbre no solo a nivel local, sino más bien a nivel nacional ya que el Ecuador se encuentra en el cinturón de Fuego de pacifico, es por esta razón que lo hace más vulnerable a sufrir amenazas derivadas de la geodinámica interna de la tierra, por lo cual mediante la metodología STAR se pude evaluar de manera detallada a la amenaza sísmica, la vulnerabilidad y por consiguiente el riesgo

Haciendo una relación entre los estudios descritos anteriormente con la presente investigación, está claro que sobre el peligro sísmico no se puede hacer nada ya que la generación de un movimiento telúrico no se puede predecir, sin embargo se puede estimar la magnitud de un sismo tomando en cuenta las fallas geológicas que atraviesan un determinado contexto en este caso la provincia Bolívar para ello, mediante la aplicación de la metodología STAR se obtuvo como resultados un nivel de peligro sísmico (amenaza) medio. Por otro lado para evitar los futuros riesgos de desastres se debe trabajar sobre la vulnerabilidad y de esta menara reducir el nivel de exposición ante un evento sísmico, el nivel de vulnerabilidad de la provincia antes mencionada es media al igual que la amenaza debido a que la mayoría de las infraestructuras a nivel cantonal y provincial son de hormigón armado, Finalmente la probabilidad de ocurrencia de un riesgo sísmico es medio ya que al emplear la formula ya establecida Amenaza*Vulnerabilidad $=$ Riesgo, arrojo un resultado de 0,57, este valor se 
encuentra dentro de los parámetros descrito por la metodología STAR, que al igual que los otros estudios se utilizó una herramienta informática (ArcGis) para procesar los datos desde un ordenador. Además, este dato producto de una investigación multidisciplinar, puede servir de base para futuros estudios de evaluación del riesgo sísmico a nivel local, provincial, nacional e internacional con el único objetivo de reducir los riesgos de desastres en el área geográfica afectada.

\section{Conclusiones.}

- Aunque no estaba contemplado a priori abordar cuestiones de sismicidad histórica, hay que conmiserar que el Ecuador se encuentra localizado en el cinturón de fuego del Océano Pacífico, al noroccidente de Sudamérica. Es uno de los países más densamente poblados de la región. La actividad sísmica es causada principalmente por la subducción de la Placa Oceánica de Nazca y la presencia de un complejo sistema de fallas activas locales. Bajo la información disponible y dada la alta peligrosidad sísmica a la que se ve expuesto el país, toma trascendencia el terremoto acaecido el 16 de abril de 2016, de magnitud 7.8, que colapsó alrededor de 40 edificios de hormigón armado, ubicados en el centro de Portoviejo, con dolorosas pérdidas de vidas y un cuantioso perjuicio económicos, merece ser incluido.

- Las fallas geológicas que inciden para estimar la amenaza de la provincia Bolívar fueron: La Maná, Quinsaloma, Calabi, Salinas, Guaranda, Montalvo, Chillanes, Pallatanga, La Candelaria y Guamote. Mediante la aplicación de la metodología STAR se ha logrado obtener el cálculo de la amenaza a nivel provincial, con un valor de 0,60 , datos que se adquirieron a partir de la multiplicación del factor $\mathrm{Z}$ y la magnitud máxima.

- El estudio se realizó en todos los cantones de la provincia Bolívar dando un valor de 0,95 que pertenece a una vulnerabilidad media, de los cuales predomina en 6 cantones una vulnerabilidad media y en el sobrante una vulnerabilidad baja (Las Naves).

- A partir de la amenaza y la vulnerabilidad se obtuvo el riesgo sísmico de la provincia Bolívar que corresponde a un valor de riesgo medio 0,57 , debido a que la mayor parte de las construcciones tienen una tipología estructural de concreto, lo cual lo hace más resistente ante sismos de una magnitud considerable.

- Por último, es digno de resaltar que en la provincia de Bolívar existen un gran número de viviendas que se encuentran en una edad que supera los 40 años, esto incrementa la vulnerabilidad estructural ya que estas edificaciones no cuentan con un mantenimiento periódico tanto estructural como en mampostería.

\section{Agradecimiento.}


Este trabajo ha sido parcialmente subvencionado ECOSP - Research Group, conformado por docentes investigadores bajo mutuo acuerdo de colaboración multidisciplinar para investigación. De este modo agradecemos a la Universidad Estatal de Bolívar (UEB), por contribuir con su infraestructura, equipos tecnológicos y laboratorios pertinentes para la recolección y procesamiento de información. Del mismo modo expresamos nuestro agradecimiento al Sr. Ronald Estrella Velasco quien colaboro, desempeñándose como estudiante de la Carrera de la carrera de Administración para desastres y Gestión de Riesgos de la UEB.

\section{Referencias bibliográficas.}

Aguilar, R., \& Mieles, Y. (2016). Análisis de los edificios que colapsaron en Portoviejo durante el terremoto del 16 de abril de 2016. Revista Internacional de Ingeniería de Estructuras, 21(3), 257-282.

Aledo, A., \& Sulaiman, S. (2015). La incuestionabilidad del riesgo: vulnerabilidad social y riesgo sísmico en municipios turísticos. Cuadernos de Turismo, 36, 17-37. doi:http://dx.doi.org/10.6018/turismo.36.230861

Álvarez, R. (2012). Metodología de la investigación: Operacionalización de Variables. Medellín. Colombia: McGraw-Hill.

Amangadi, C. E., Yasuma, L. E., \& Barragán, A. G. (2019). Análisis de riesgos ante eventos sísmicos en las edificaciones de la parroquia Santa Fe, cantón Guaranda, provincia Bolívar, Ecuador. Revista de Ciencias de Seguridad y Defensa, IV(3), 50-88.

Ayala, O. R., Delgadillo, S. A., \& Ferrer, O. C. (2017). Amenaza sísmica en Latinoamérica. Revista Geográfica Venezolana, 58(2), 258-262.

Beauval, C., Yepes, H., Palacios, P., Segovia, M., Alvarado, A., Font, Y., . . Vaca, S. (2013). An Earthquake Catalog for Seismic Hazard Assessment in Ecuador", Bulletin of the Seismological Society of America. Bulletin of the Seismological Society of America, 103(2A), 773-786. doi:10.1785/0120120270

Benavides, B. M. (2018). Reubicación de la población ante eventos sísmicos en el valle del río San Pedro. Estado Miranda, Venezuela. Terra Nueva Etapa, XXXIV(56), 1-26.

Carrión, A., Giunta, I., Mancero, A., \& Jiménez, G. (2017). Posterremoto, gestión de riesgos $y$ cooperación internacional. Quito: Editorial IAEN. Obtenido de https://www.preventionweb.net/files/57529_posterremotov6final.pdf

Chunga, K. (2016). Geología de Terremotos y Tsunami. Quito: Sección Nacional del Ecuador del Instituto Panamericano de Geografía e Historia, IPGH. 
Contreras, L. M. (2014). Riesgo de Tsunami en Ecuador. 68-75 : Ingenius. Revista de Ciencia y Tecnología, 12.

Díaz, F. D. (2017). Un método simplificado para evaluar el riesgo sísmico y priorizar la atención de los bienesculturales inmuebles: el caso de Chile. Intervención, Revista Internacional de Conservación, Restauración y Museología, 8(15), 46-62.

Eguez, A. (2013). Database and Map of Quaternary faults and folds of Ecuador and its offshore regions. Report 2003-289 (Inglés). Golden: The United States Geological Survey (USGS) and U.S. Department of the Interior.

Fernández, G. (2017). Propuesta metodológica: construcción de un índice de vulnerabilidad global ante la amenaza sísmica, en espacios regionales, con el uso de técnicas estadísticas multivariables. Terra Nueva Etapa, XXXIII(54), 127-163.

Jiménez, P. J., Cabrera, C. J., Sánchez, B. J., \& Avilés, T. F. (2018). Vulnerabilidad sísmica del patrimonio edificado del Centro Histórico de la Ciudad de Cuenca: Lineamientos generales y avances del proyecto. MASKANA, 9(1), 59-78. doi:10.18537/mskn.09.01.07

Martínez, V. E. (2018). Inteligencia geoespacial para desastres sísmicos: investigaciones en CentroGeo. Revista mexicana de sociología, 80, Núm. Especial, 71-94. doi:10.22201/iis.01882503p.2018.0.57774

Montiel, A. K., \& Negrete, M. Á. (2017). Gestión del riesgo sísmico en el estado Zulia: Una propuesta desde la educación geográfica. Omnia, 23(2), 67-79.

Ordoñez, B. A. (2016). Evaluación de la vulnerabilidad sísmica de las edificaciones de la ciudad de Quito - Ecuador y riesgo de pérdida. Quito: Escuela Politécnica Nacional. Recuperado el 01 de 08 de 2019, de https://bibdigital.epn.edu.ec/handle/15000/16537

Porras, J. L., Linkimer, L., Arroyo, I. G., \& Taylor, M. (2018). Actividad sísmica percibida en costa rica durante el 2017. ctividad sísmica percibida en costa rica durante el 2017, 203-214. doi:10.15517/rgac.v58i0.32852

Quinde, M. P., \& Reinoso, A. E. (2016). Estudio de peligro sísmico de Ecuador y propuesta de espectros de diseño para la Ciudad de Cuenca. Revista de Ingeniería Sísmica, 94, $1-26$.

Salgado-Gálvez, M. A., Carreño, M. L., Barbat, A. H., \& Cardona, O. D. (2016). Evaluación probabilista del riesgo sísmico en Lorca mediante simulaciones de escenarios. Revista Internacional de Métodos Numéricos para Cálculo y Diseño en Ingeniería, 32(2), 7078. doi:https://doi.org/10.1016/j.rimni.2014.12.001 
Vidaud-Quintana, I. N., Frómeta-Salas, Z. P., \& Vidaud-Quintana, E. d. (2019). Control de calidad en estructuras de hormigón para la construcción en zonas de elevado peligro sísmico. Ciencia en su PC, 1(4), 72-85.

Zambrano-Hernández, L. A., \& Gómez-Serna, E. A. (2015). Prácticas culturales y gestión del riesgo sísmico: la cultura de las buenas costumbres. Revista Facultad Nacional de Salud Pública, 33(3), 388-396. doi:10.17533/udea.rfnsp.v33n3a08

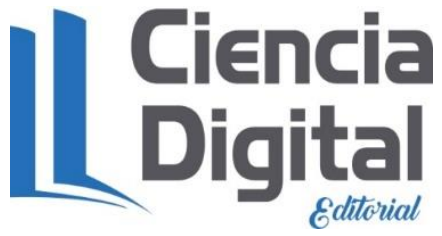


PARA CITAR EL ARTÍCULO INDEXADO.

Serrano Castro, A. K., Muyulema Allaica, J. C., Usca Veloz, R. B., \& González Rivera, M. M. (2019). Aplicación de la metodología STAR para el análisis del riesgo sísmico de la provincia Bolívar Ecuador. Ciencia Digital, 3(4), 227-249. https://doi.org/10.33262/cienciadigital.v3i4.968

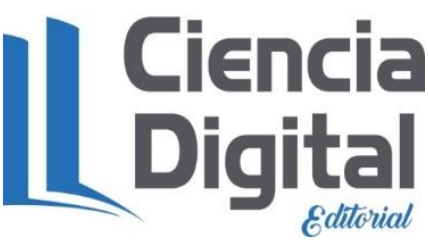

El artículo que se publica es de exclusiva responsabilidad de los autores y no necesariamente reflejan el pensamiento de la Revista Ciencia Digital.

El artículo queda en propiedad de la revista y, por tanto, su publicación parcial y/o total en otro medio tiene que ser autorizado por el director de la Revista Ciencia Digital.
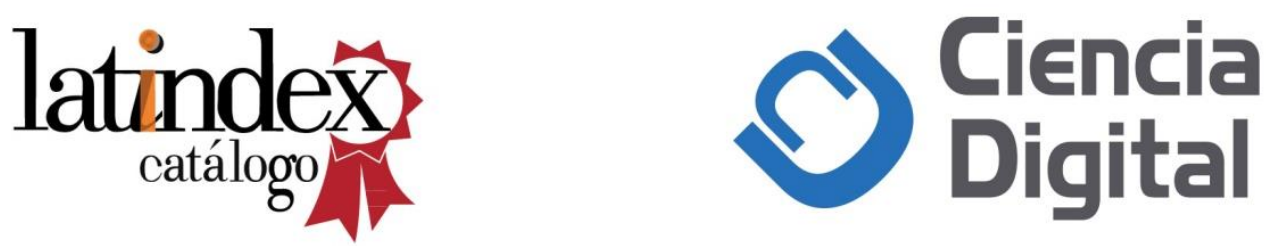